\title{
DEVELOPMENT OF A VIRTUAL METERING SYSTEM APPLICATION FOR HIGH WATER CUT WELLS WITH ELECTRIC SUBMERSIBLE PUMPS (ESPS) - A CASE STUDY FOR QUIFA FIELD
}

\author{
Miguel Asuaje ${ }^{1 *}$, Julieth Zapata ${ }^{1}$, and Wilmer Arcos ${ }^{1}$ \\ ${ }^{1}$ Frontera Energy, Operations Technology Department, Calle 110 \# 9 - 25 Bogota, Colombia
}

\begin{abstract}
Well surveillance is a mandatory requirement for reservoir characterization and production optimization that every asset must aim for. To guarantee constant monitoring of a single well, installing conventional multi-phases flow meters systems is necessary. As an alternative way, this paper presents a Virtual Metering System (VMS) developed to estimate flow rates in oil wells with high water cut operating with electric submersible pumps as artificial lift systems. The VMS starts from a mathematical model based on production variables, well data, and pump performance curves to estimate fluids flow rates and water cut using a Newton Raphson Multivariate Method. The VMS was implemented in 11 wells in Quifa Field, Colombia. The VMS is constantly feeding by data from the PI System. The VMS results show a deviation of less than $3 \%$ in calculating the fluid flow rate and less than $5 \%$ in estimating the water cut.
\end{abstract}

\section{Introduction}

The continuous monitoring of the production variables of a well, such as the fluid flow rates and the water cut, is of great importance for the reservoir's characterization and production optimization. These activities are the key to maximizing production, oil recovery, and reducing operating costs. Monitoring the production behavior and forecast of each well (water cut, GOR, total fluids) depends on operational variables (shock opening, pump frequency). Monitoring is done by installing a flow meter system, generally using separators or test lines. In the operation of onshore heavy oil fields characterized by a low GOR and high water cuts, a common technique to measure fluid production is to take a sample at the wellhead and make a laboratory analysis. Unfortunately, under this methodology, water cut measurements keep a high degree of uncertainty due to technical and human factors in the sampling procedure. Besides, the possibility of taking samples with an acceptable periodicity becomes almost impossible due to the high number of samples taken per day and the limited capacity of the field laboratories.

One way to mitigate or reduce the uncertainty associated with the measurement technique described above would be the physical installation of multiphase meters in each well. However, in the existing technologies, the discrimination of liquid phases results in significant uncertainties. On the other hand, the costs associated with this measurement methodology would be very high, making the implementation impossible for each well in the field.
Computational power growth, network, and communication systems have allowed the development of much cheaper alternatives to monitor and gather information required to improve oil fields' development. During the last years, virtual metering has emerged as an alternative to have input in real-time and is revolutionizing the way to manage the oil fields. These virtual measurement systems allow obtaining/inferring each phase's flow rates from operational and process variables, which are always available in the information systems (SCADA, PI, etc.) of the fields. With a certain margin of uncertainty, the virtual meters allow a very close approximation to the actual conditions depending on the field's amount of historical data and the tuning process's effectiveness.

\section{Multiphase measurement}

A multiphase flowmeter is a device capable of determining the volumetric rates of each component from a multiphase mixture [1]. Multiphase flow meters can be classified as follows (Figure 1).

\footnotetext{
* Corresponding author: asuajem@,fronteraenergy.ca
} 


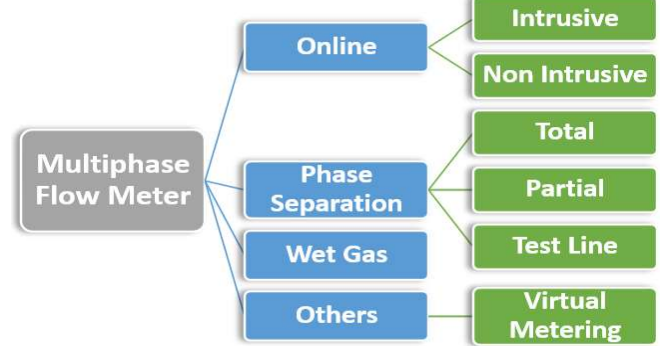

Fig. 1. Classification of multiphase flowmeters.

Since the 1980s, the traditional way of measuring using test separators has evolved using multiphase meters toward virtual meters (Figure 2).

This development came in response to the need for the oil industry to have new technologies that facilitate offshore operations, replace traditional test separators, and avoid lines and test manifolds. All this would result in saving space, weight, time, and money in the production platforms

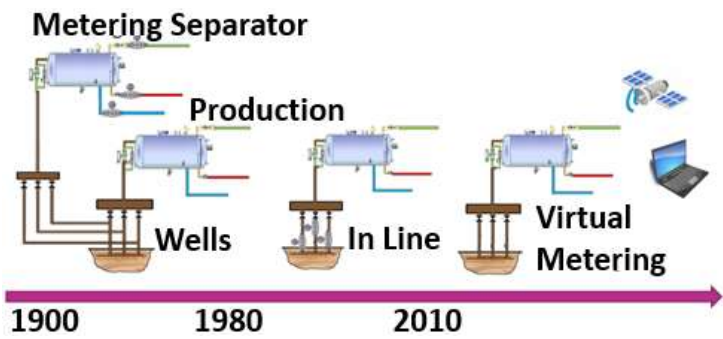

Fig. 2. Schematic of a reservoir development: a) using test separator b) using multiphase meters online, c) Virtual Measurement.

The use of online multiphase flowmeters has helped to solve specific needs in four different areas of the oil industry: (a) allows the implementation of a continuous (but not simultaneous) monitoring of each well, which could help in the designing of more effective exploitation plans and the increase of the recovery factor of the reservoir; (b) allows the production of various fields to be mixed even if they belonged to different companies, determining with certainty what percentage of the total production corresponds to each owner; (c) helps in the process control by knowing the effect of secondary recovery techniques applied, and (d) facilitates the process of inspection of production for purposes of tax payments and corresponding duties. Although many online multiphase measurement systems have been developed, no reference can be made to any of them as a general or universally accurate application. The requirements that must be met in terms of accuracy are different for each country, and the range of acceptance will depend on the final measurement purpose. Therefore, different levels of accuracy may be acceptable. For reservoir management and optimization of the production, accuracy between 5 and $10 \%$ could be accepted. [2] .

Today, virtual flow meters have become the good natural evolution of inline multiphase flow measurement as the new technical alternative for monitoring and optimizing production. Virtual measurement systems allow the estimation of total flowrates and phase fractions based on the analysis of operational variables monitored online such as pressures, temperature, and operating parameters of the artificial lift equipment. These variables, obtained from operational data, can be processed using analytical equations or from any system of pattern recognition or statistical signal processing. Also, for some cases, virtual measurement systems have been developed based on simulation programs, which allow the determination of some variables that are not available in the field using correlations.

Virtual measurement systems are equipped with realtime calibration programs that reunite the total production of wells, daily or weekly, with the fiscal production. Then the back rate allocation is assigned to the wells according to each flow rate's calculated uncertainties.

Large companies such as Shell, Forsys Subsea, FMC, Statoil (Equinor) have implemented virtual measurement systems (VMS) in some of their wells and fields. In their works, they show savings in:

1.Completion System. Savings in CAPEX increases linearly with the number of installations in the wells of the field.

2.Control System. Without a flow meter installed, the equipment needed for the meter gets lower.

3.Installation. Without the installation of a physical flow meter, savings in time and marine travel are crucial for Offshore platforms

Additionally, they report substantial operational improvements in:

1. Less well test frequency.

2. Better ability to increase production.

3. Depending on the level of uncertainty that is required, a virtual meter is installed without any extra measurement (Physical Meter) with uncertainties less than $10 \%$.

4. It allows for new wells to identify significant fluid phase changes (e.g., large increase in water cut).

5. Simultaneous and continuous wells monitoring.

In 2005, Dellarole et al. [3] installed a virtual measurement system based on pressure and temperature signals, throttling valves upstream and downstream, and signals from pressure and temperature along the production line. The VFM provides accurate estimates of gas and liquid flow rates. Their method can be applied to critical and subcritical flows and is maintained for a wide range of flow conditions (choke geometry, fluid properties, GVF, etc.). The authors present the VMF application results for various fluids (light oil, light oil with high GOR, medium oil, medium oil with high GOR, and condensed gas) in several fields. In general, the reported error is less than $10 \%$.

Haldipur et al. [4] analyzed advances in virtual measurement technology. They presented the experience obtained in the Gulf of Mexico and several other places worldwide. The best project execution practices, including specifications, acceptance tests, and start-up procedures, are discussed. The document presents a comparison with field data. It shows that this technology has provided very reliable and accurate flow predictions on a variety of wells configurations and 
reservoir characteristics (from gas condensate to black oil systems in a wide range of GOR / GLR). They suggest recalibrations every month, whose factors should be between 2 and $3 \%$.

Garcia et al. [5] managed to generate an alternative to measuring the wells' production. The author described the methodology for data selection, analysis, and validation of sensors, modeling, online implementation, and quality control. Comparing the production rate estimated by the method against the audited one, errors less than $4 \%$ are obtained.

Petukhov et al. [6] to calculate the oil, water, and gas rates in the offshore field of Equatorial Guinea, called Ceiba, used a methodology to validate the data combining the uncertainty and the current models to minimize the error of the global function. The project's benefits include the reduction, up to $65 \%$, of the time implemented to validate tests and to allocate the wells to wells production.

Hauoche et al. [7] generated a methodology that models the ESP's performance with a virtual meter. This new method considers the effect of the gas production and reports the result of three cases applied; for a well with pump inlet conditions above the bubble point, for a well with pump inlet below the bubble point, and another with the two previous states grouped.

$\mathrm{Ma}, \mathrm{X}$. [8] developed a production monitoring system and an optimization system that works in real-time, where the optimizer integrates data available from monitoring. This method was used for wells that produce commingled and a regression algorithm capable of adapting to changes in the well's conditions. This technology was applied in an offshore asset, and early benefits have been obtained in terms of a $2 \%$ increase in production from the optimizer's suggestions.

\subsection{Problem description}

Quifa is one of the leading heavy oil reservoirs in Colombia. It was developed by drilling horizontal and deviated wells and taking advantage of a very active aquifer and high permeability sands. These conditions make it possible to have a total fluid production of 1,320 KBPD with $96.7 \%$ of water cut through 272 active wells, where more than $80 \%$ have electro submersible pumps (ESP) as artificial lift system. Surface trunk lines follow an irregular topography over large distances, creating significant hydraulic imbalances and restricting the processing facility's inlet flow. These conditions have caused a very high energy consumption and intense competition between wells in each cluster and the trunk lines.

An optimization methodology has been developed To guarantee efficient exploitation of a reservoir of this size by mitigating these bottlenecks. This methodology uses fluid simulation and optimization software from the well to the fluid processing center.

The model is updated and tuned daily using the field data introduced to the simulator and the optimizer to obtain the operative recommendations that guarantee the maximum oil production considering water and energy restrictions. [9].
Nevertheless, one of the improvable aspects of the optimization routine is the amount of data and the frequency in which the wells are sampled to determine their flow rate and water cut. Both parameters are required to be known in real-time to find optimization opportunities.

In this sense, virtual measurement systems rise as an alternative to monitor $24 / 7$ the most significant number of wells in the field, in real-time and simultaneously. Another advantage of this system is the low investment and the ability to use already available data.

\subsection{Virtual metering system}

The virtual measurement system uses a mathematical model based on three equations which are:

1. Equation of pressure loss in the tubing

2. Pump curve

3. Pump power

These three equations can be written as a function of the three unknown variables. The water cut of the mixture strongly influences these variables. These variables are: 1. Total fluid flow rate.

2. Water cut

3. Pump power.

The general scheme of resolution is presented in figure 3.

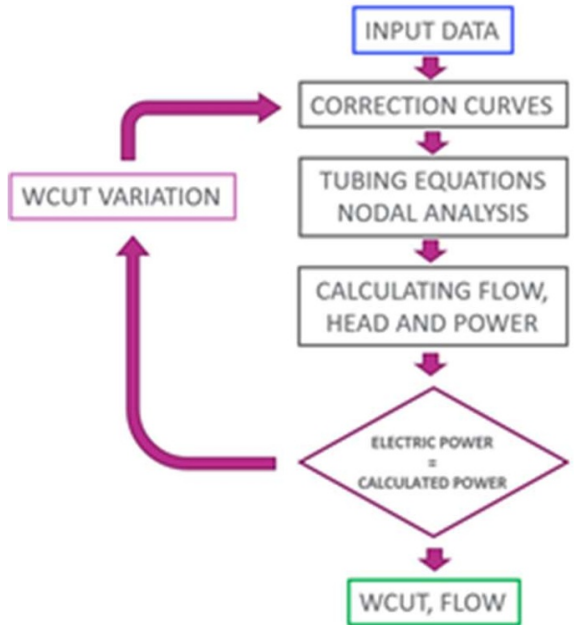

Fig. 3. Virtual Measurement Process

For each well, its completion diagram and the pump's performance curve are needed (figure 4) to extract information like depths, internal diameters, and the pump's operating characteristics. 


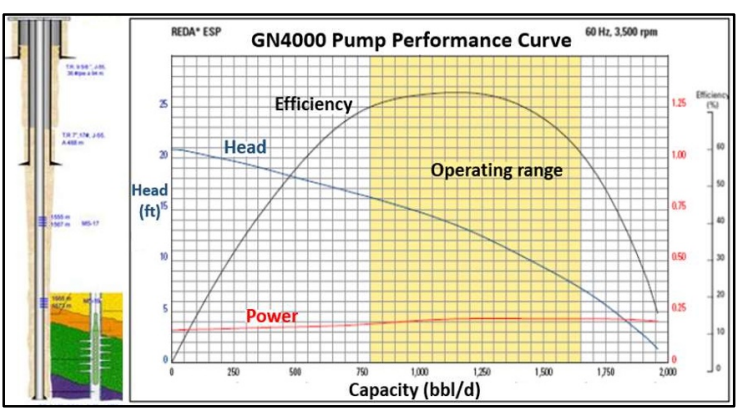

Fig. 4. Mechanical State and curve of the ElectroSubmersible Pump.

The Virtual Meter requires minimum information to operate, such as the tubing's internal diameter (ID), depths of the sensors, and other information contained in the mechanical state; pump operating curve, the power consumed by the motor and production data as fluid, system pressures and pump frequency.

All equations should be adjusted and corrected to each well's performance, as indicated in Figure 5.

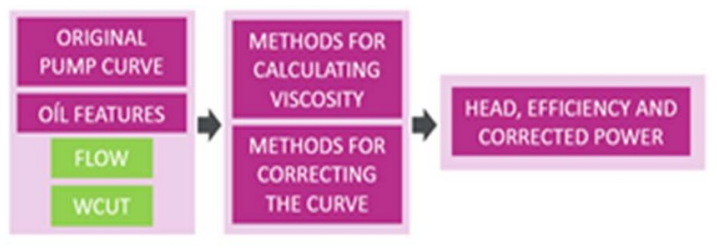

Fig. 5. Process of correction of the curve of the pump

\subsubsection{Pump equation}

The best polynomial fit equation is found for each performance curve. The centrifugal pump's performance will be affected by the fluid viscosity, which is a function of the mixture water cut (oil-water).

Centrifugal pump performance's curves must be corrected following this procedure:

1. Find the viscosity of the mixture depending on the water cut

2. Estimate correction coefficients of the pump curve performance as a function of fluid viscosity.

3. Correction by speed rotation or frequency.

\subsubsection{Mixture viscosity}

Normally, oil viscosity is higher than water viscosity, and for an oil-water mixture, an inverse relationship between water cut and mixture viscosity is typical.

The viscosity of the mixture can be determined employing one of these three methods:

Refutas method (1947)

Method of Kendall \& Monroe (1917)

Method of Barrufet \& Setiadarma (2003)

These methods are based on table A-1, "Summary of Common Viscosity Blending Techniques," [10]. The methods do not consider two effects that can occur in the mixture between oil and water. The first is the effect of the emulsion, and the second is the point of inversion.
The viscosity of the Quifa oil and water mixture is shown in Figure 6. The mixture's viscosity decreases as the water cut increases.

The Refutas method was retained because it offers a better adjustment and stability in solving the equation system.

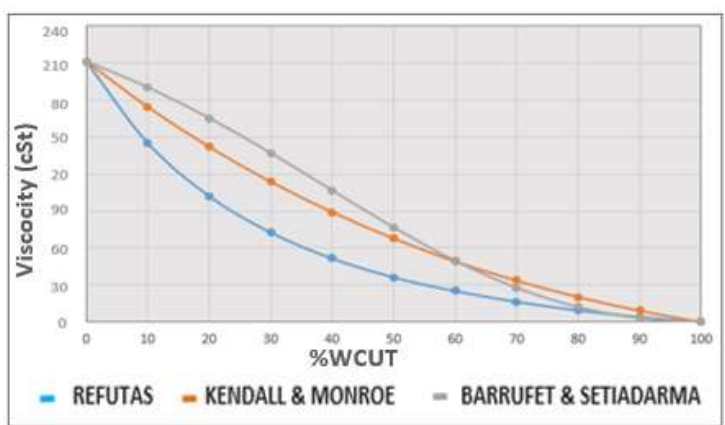

Fig. 6. The viscosity of the Quifa oil-water flow mixture

\subsubsection{Viscosity and variable speed Effect}

The virtual metering system requires the correction of the pump's performance curve by handling a multiphase flow. Chances on the viscosity of the mixture will have an impact on the performance of the pump.

Three methodologies were studied to correct the performance of the pump due to the viscosity effect. These are:

Turzo et al. [11]

Gunnar Hole. [12]

Hydraulic Institute. [13]

These methods have been developed from empirical equations representing the behavior of the graphics proposed by the Hydraulic Institute. For virtual metering, the Turzo model offered a simplified way to correct performance curves. The performance of pumps at different speeds is well known, and basic operation rules have been established. The relationships between the performance parameters (capacity, head, efficiency, and power) and the pump's current rotational speed can be estimated according to affinity laws [14].

\subsubsection{Equation of Pipeline Losses}

The pump's discharge pressure can be estimated by performing a wellhead's nodal analysis. An adjusted Bernoulli equation can evaluate the pipeline's energy losses. It should be considered the multiphase flow as a homogeneous mixture.

\subsubsection{Equation of Electric Power}

The drive power of a hydraulic pump can be expressed as the product of the power supplied to the fluid by its flow rate, gravity, and the mixture's density. All these parameters result from the water cut and the flow rate. 


\subsubsection{Solution}

The equations described in Figure 7 are used to perform the nodal analysis and the verification of the energy consumption of the pump,

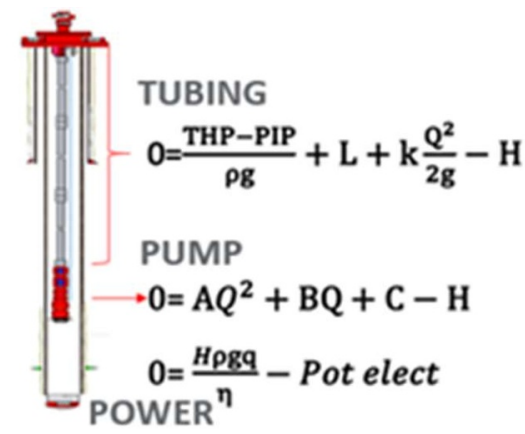

Fig. 7. Equation's measurement system

The corrected equations are solved using a Newton Raphson method, obtaining a value for the pump's total flow rate, water cut, and power, up to an error of less than $1 \%$, for total flowrate, is obtained.

\section{Pilot test}

The final pilot test was conducted over eleven wells of the Quifa field with ESP and high water production. Of these eleven wells, nine showed a good agreement. The other two show deviations more significant than $10 \%$ or could not tune because of the amount and quality of the original data.

The initial VMS was implemented in Excel VBA platform, which allowed better management of production history. Initially, for each well, data was taken twice a day for three months. This original data was used to tune the model. A spreadsheet as a user interface was developed that allows data loading and execution calculations.

The calculation was carried out Off-Line, taking the production history, of three months, for each well and thus adjusting the model. As soon as the production history is entered, the flow rate and electric power calculation are compared with the reported power value. If there is a difference more significant than $1 \%$ between these two variables, a new water cut value is entered. All calculations are done again until the power related to the quantity of water in the mixture is equal to the one measured.

\section{Results}

Next, the graphs resulting from the application of the model for 1 well are shown.

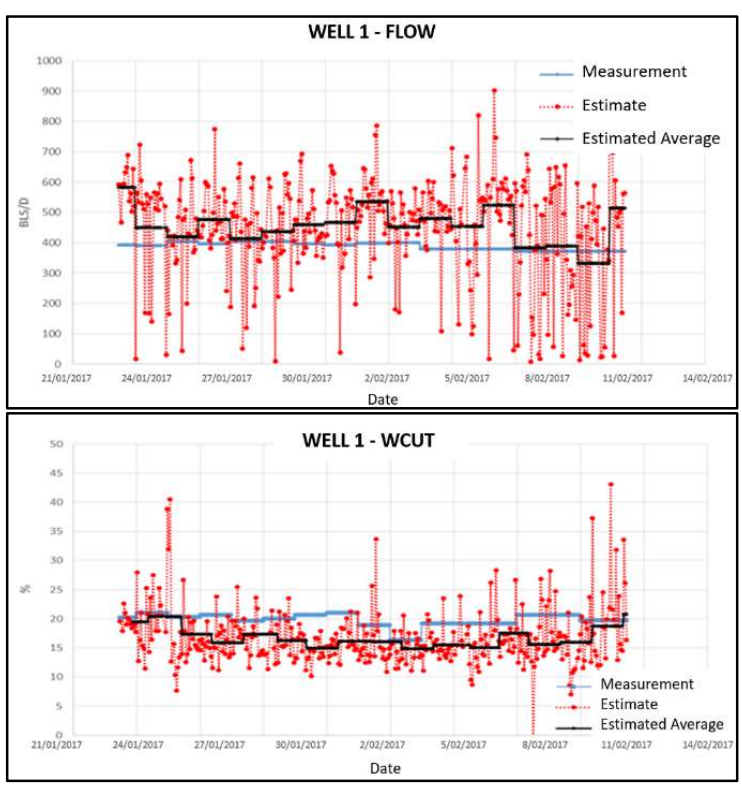

Fig. 8. The viscosity of the Quifa oil-water flow mixture

A deviation lower than $3 \%$ and $5 \%$ was obtained for total flow rate and water cut, respectively. This precision is enough for the monitoring and optimization of the wells.

Results for well 1 are shown in figure 8. VM results seem to be more realistic concerning the fluctuating and unstable multiphase flow behavior expected into the well that the field's test results reported. Real-time monitoring is one of the benefits of a virtual metering system. It allows estimating the flow and cut-off values of water at any operation time, unlike the current measurement method where the flow rate and water cut are determined from well test procedure performed, in the best case, one time a week.

Sources of uncertainty or deviations in the VMS values are flow patterns, pump wear, interference with other wells. These drawbacks can be minimized by adjusting the model before the measurement using each well's production history.

This calibration is performed iteratively, looking for an equation describing the fluid's behavior and corresponding to reported power consumption. This adjustment process must be carried out each time the well is intervened when the ALS is altered or when the behavior of reservoir fluids changes drastically.

\subsection{Web Implementation of the VMS}

The first model was migrated to a WEB platform that feeds the wells' SCADA data. The update period in the estimation of flow rate and water cut is four minutes. The results are published graphically, comparing the data measured in the field (Figure 10). 


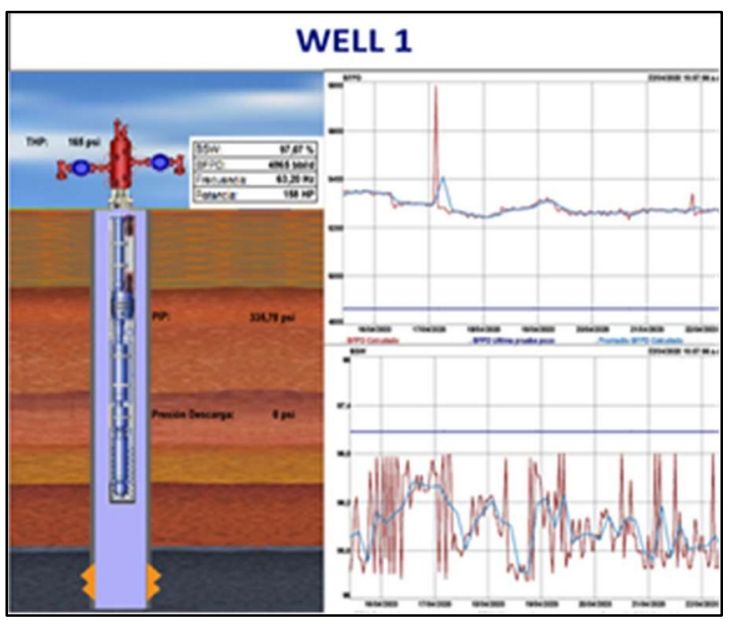

Fig.9. WEB interface for the virtual meter.

To track the tool, one can click on any variable and visualize its behavior in the requested period, as shown in figure 11, for the flow rate and the water cut, respectively.

The results obtained, with the automatic VMS, show an error lower than 7\%. However, the increase in data shows peaks probably due to instantaneous fluctuations in the measurement systems. The availability, sampling frequency, and quality of the measurements as input data will impact the VMS's estimation. Therefore, some filters must be applied to obtain values in an acceptable range.

Nevertheless, with an error being minor than $10 \%$, results are acceptable for monitoring and optimizing production.

\section{Conclusions}

1. A virtual metering system was built for wells with high water cuts and electro submersible pumps. Once the model is tuned, the results show a good agreement with total flowrate measurements a water cut.

2. For a primary VMS, an Excel platform was used and validated over 11 wells in the Quifa field in Colombia 3. After making the corresponding adjustment to the well, the program can perform virtual flow and water cut measurements in real-time with low uncertainty level; $<3 \%$ in fluid rate, and $<5 \%$ in water cut. However, the model must be tuned periodically to obtain calculations very close to reality during the well's life.

4. It was observed that for wells with low water cut $(<30 \%)$, the uncertainty increases considerably. Most likely, this is due to the effect of the viscosity correction, which for this range may not work correctly.

5. A real-time integrated Virtual metering system was build using a web platform and the remote connection to the SCADA information systems.

6. With this system, wells can be monitored $24 / 7$ simultaneously.
7. Due to the increase of the amount of data (without using a filter), error in flowrate and water cut estimation increased by $7 \%$. Nevertheless, these results are still acceptable to monitoring and optimizing well performance.

\section{Referencias}

1. S. Corneliussen, J. Couput, E. Dahl and E. Dykestee, "Handbook of multiphase Flow Metering," (2007).

2. G. Falcone, G. Hewitt y C. Alimonti, Multiphase Flow Metering: Current Trends and Future Developments, (2002).

3. E. B. Dellarole, Virtual Metering And Flow Allocation: Models, Tools And Field Results. Offshore Mediterranean Conference, Ravenna Italy, (2005).

4. P. Haldipur, « Virtual Metering Technology Field Experience Examples. Offshore Technology Conference,» (2008).

5. A. A. Garcia, An Implementation of On-line Well Virtual Metering of Oil Production. SPE Intelligent Energy Conference and Exhibition, 23-25 March,. Utrecht, The Netherlands: Society of Petroleum Engineers., (2010).

6. A. Y. Petukhov, Virtual Metering System Application in the Ceiba Field, Offshore Equatorial Guinea, Texas, USA, (2011).

7. M. Haouche, A. Tessier y Y. Deffous, «Virtual Flow Meter pilot: based on Data Validation and Reconciliation Approach.,» SPE International Production and Operations Conference \& Exhibition, (2012).

8. M. Xiang, B. Zachary y P. Porto, «Real-Time Production Surveillance and Optimization at a Mature Subsea Asset.,» SPE Intelligent Energy International Conference and Exhibition, (2016).

9. M. Asuaje, Improved Production using Simulation and Optimization Softwares in Quifa Field, Colombia, Bogotá, Colombia: ACIPET Annual Conference, (2017).

10. R. Sutton y D. Bergman, Application of Bergman-Sutton Method for Determining Blend Viscosity, SPE Prodnction \& Operation, (2012).

11. Z. Turzo, G. Takacs y J. Zsuga, Equations Correct Centrifugal Pump Curves for Viscosity., Oil \& Gas Jurnal, (2000).

12. G. Hole, «Fluid Viscosity Effects on Centrifugal Pumps.,» Pump and Systems magazine, (2010).

13. Hydraulic Institute, Determination of Pump Performance when Handling Viscous Liquid. Hydraulic Institute Standards, 20th Ed, (1969).

14. A. Stepanoff, Centrifugal and Axial Flow Pumps. Theory, Design, and Application, New York: John Wiley \& Sons, Inc., (1957). 